\title{
Early initiation of combined antiretroviral therapy preserves immune function in the gut of HIV-infected patients
}

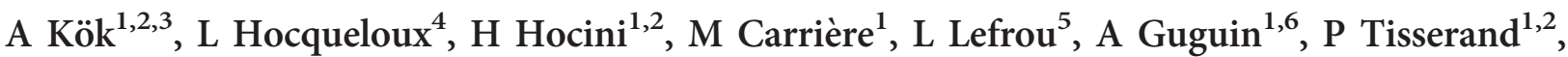 \\ H Bonnabau ${ }^{2,7}$, V Avettand-Fenoel ${ }^{8}$, T Prazuck ${ }^{4}$, S Katsahian ${ }^{9}$, P Gaulard ${ }^{3,10,11}$, R Thiébaut ${ }^{2,7}$, \\ Y Lévy ${ }^{1,2,3,12,14}$ and S Hüe ${ }^{1,2,3,13,14}$
}

\begin{abstract}
Massive loss of lamina propria CD4 ${ }^{+} \mathrm{T}$ cells, changes in the lymphatic architecture, and altered intestinal epithelial barrier leading to microbial translocation are the common features of HIV-1 infection and are not fully restored under combined antiretroviral therapy (CART). To better understand determinants of gut mucosal restoration, we have performed phenotypic and gene expression analyses of the gut from HIV-infected patients, naive or treated with cART initiated either at the early phase of the primary infection or later during the chronic phase. We found a depletion of T helper type 22 (Th22) and interleukin-17-producing cells in naive patients. These populations, except Th22 cells, were not restored under CART. Regulatory T cells/Th17 ratio was significantly increased in HIV-infected patients and was inversely correlated to the restoration of $\mathrm{CD} 4{ }^{+} \mathrm{T}$ cells but not to gut HIV DNA levels. Gene profile analysis of gut mucosal distinguished two groups of patients, which fitted with the timing of cART initiation. In their majority early, but not later treated patients, exhibited conserved intestinal lymphoid structure, epithelial barrier integrity and dendritic cell maturation pathways. Our data demonstrate that early initiation of CART helps to preserve and/or restore lymphoid gut mucosal homeostasis and provide a rationale for initiating cART during the acute phase of HIV infection.
\end{abstract}

\section{INTRODUCTION}

$\mathrm{CD}^{+} \mathrm{T}$ cells in the gut-associated lymphoid tissue (GALT) are depleted early after HIV and Siminan immunodeficiency virus (SIV) infections. ${ }^{1-3}$ This depletion is associated with an apoptosis of enterocytes and an alteration of the structural intestinal barrier. ${ }^{1-3}$ These damages result in an increase of intestinal permeability and facilitate microbial translocation, which likely contribute to chronic immune activation and disease progression. ${ }^{4}$

Another feature of pathogenic HIV and SIV infections is the preferential loss of mucosal T helper type 17 (Th17) cells, ${ }^{5,6}$ which contributes to the damage of the epithelial barrier. ${ }^{7}$
These cells are enriched in the intestinal lamina propria and have a critical role in defense and homeostasis. Besides Th17 cells, other lymphoid populations producing interleukin (IL)-17 have been described in the GALT such as T-cell receptor (TCR) $\gamma \delta$ expressing T cells ( $\gamma \delta \mathrm{T}$ cells), innate immune lymphocytes (ILC) $)^{8,9}$ and mucosal-associated invariant T (MAIT) cells, which can rapidly respond to pathogens by secretion of IL-17 in an innate-like manner. ${ }^{10}$ These cells produce IL-17 and IL-22, two cytokines that stimulate the production of antimicrobial proteins by the epithelium and thereby sustain its barrier function. ${ }^{11}$ IL-17 also induces the recruitment of neutrophils that eliminate bacteria having

\footnotetext{
${ }^{1}$ INSERM U955, Team 16, Créteil, France. ${ }^{2}$ Vaccine Research Institute (VRI), Université Paris Est Créteil, Faculté de Médecine, Créteil, France. ${ }^{3}$ Université Paris Est Créteil, Faculté de Médecine, Créteil, France. ${ }^{4}$ Service des Maladies Infectieuses et Tropicales, CHR d'Orléans-La Source, Orléans, France. ${ }^{5}$ Service d'Hépato-Gastro-Entérologie, CHR d'Orléans-La Source, Orléans, France. ${ }^{6}$ Plateforme de Cytométrie en flux, IMRB, UFR de Médecine, Créteil, France. ${ }^{7}$ INSERM U897 - INRIA SISTM - Univ. Bordeaux Segalen ISPED, Bordeaux, France. ${ }^{8}$ AP-HP, CHU Necker-Enfants Malades, Laboratoire de Virologie, Paris, France. ${ }^{9}$ Assistance publique-Hôpitaux de Paris (AP-HP)-Hôpital Henri Mondor-Université Paris-Est Créteil, Val-de Marne, France. ${ }^{10}$ INSERM U955, Team 9, Créteil, France. ${ }^{11}$ Département de Pathologie, Groupe Hospitalier Henri Mondor, AP-HP, Créteil, France. ${ }^{12}$ Service d'Immunologie Clinique, Groupe Hospitalier Henri Mondor, AP-HP, Créteil, France and ${ }^{13}$ Service d'Immunologie Biologique, Groupe Hospitalier Henri Mondor, AP-HP, Créteil, France. Correspondence: Y Lévy or S Hüe (yves.levy@hmn.aphp.fr or sophie.hue@hmn.aphp.fr)

${ }^{14}$ Co-authors.
} 
translocated across epithelium. It has been shown that ILC-17, MAIT, and $\gamma \delta \mathrm{T}$ cells are depleted in SIV-infected macaques. ${ }^{6}$ However, whether the frequency or phenotype of these cells are perturbed in HIV-infected individuals remains unknown.

As shown in several models, if left unchecked, Th17 cells can provoke inflammation and tissue destruction. ${ }^{12}$ This effect of Th17 cells is balanced by regulatory T cells (Treg), which are accumulated in the lamina propria of SIV-and HIV-1-infected mucosal tissues. During HIV-1 infection, the ratio of Th17 to Treg is decreased in both peripheral blood and rectosigmoid biopsies. It has been suggested that a dysbalance of the Th17/ Treg ratio may reflect the maintenance of a chronic inflammatory state and is a strong predictor of SIV/HIV infection disease progression. ${ }^{12-14}$

Studies have provided a general consensus in pathogenic characteristics of T-cell depletion in HIV- and SIV-pathogenicinfected mucosal tissues. However, one important question remains whether combined antiretroviral therapy (cART) can completely restore GALT homeostasis. Although, cART has significantly improved the prognosis of HIV-infected individuals, they remain at increased risk of morbidity and mortality owing to diseases, such as cardiovascular disease, malignancy, and osteoporosis. ${ }^{15}$ These clinical events are supposed to be related to residual immune activation that persists and the incomplete immune reconstitution in the gut and in the blood in cART-treated patients. ${ }^{16}$ Recently, the attention has turned toward the long-term benefits of treatment initiated during the primary phase of infection. Starting cART as soon as during the primary phase of infection may impact biological parameters, such as depletion of viral reservoirs and immune restoration. ${ }^{17}$ Moreover, some patients who have interrupted cART initiated during primary infection were shown to be able to control their viral load and maintain low level of HIV-1 viral reservoir as well as their immune function. ${ }^{18,19}$

Therefore, these observations raise the question whether the timing of cART initiation may impact differently the restoration of gut homeostasis. By comparing the phenotype of Th17, Treg, and innate cells and the gene expression profile in gut mucosa of HIV-infected patients naive or treated with cART initiated either at the early phase of the primary infection (early cART) or later during the chronic phase (late cART), we begin to provide answer to this challenging question.

\section{RESULTS}

\section{Study participants}

Paired peripheral blood mononuclear cells and rectal biopsies were collected on the same day from $33 \mathrm{HIV}$-1-infected individuals either naive of antiviral therapy (naive cART $\mathrm{HIV}+; n=6$ ) or under long-term effective ART (cART + $\mathrm{HIV}+; n=27$ ). This later group included patients who started cART within 4 months after the diagnosis of primary HIV infection (early cART; $n=12$ ) defined by a negative or weakly positive enzyme-linked immunosorbent assay (ELISA) with incomplete HIV-1 western blotting ( $\leqslant 4$ bands) and positive plasma viral load and/or a positive HIV ELISA following a negative ELISA within the preceding 3 months. The second group of treated patients started cART later, i.e., during the chronic phase (i.e., Fiebig VI) ${ }^{20}$ of HIV infection (late cART; $n=15)$. As controls, gut biopsies from seven HIV-1 seronegative individuals (HIV - ) who underwent colonic surgery for cancer were studied. All rectal biopsies (around 10 punches for each patient and approximately $2 \mu \mathrm{l}$ for each punch) were performed at the same site (i.e., at $10-15 \mathrm{~cm}$ from the anal margin) to avoid potential regional variation among patients. The local Ethics committee (Tours, France) approved this study. All patients gave written informed consent. Characteristics of HIV-1-infected patients are summarized in Table 1.

\section{$\mathrm{CD}^{+}{ }^{+} \mathrm{T}$ cells and epithelial integrity remain incomplete in the gut of HIV-1-treated patients}

Flow cytometry analysis on freshly isolated cells from the rectal mucosa showed a significant decrease in the frequency of $\mathrm{CD} 4{ }^{+} \mathrm{T}$ cells in naive cART HIV + individuals as compared with seronegative individuals $(26.0 \pm 6.5 \%$ vs. $56.2 \pm 3.0 \%$, respectively, $P<0.01)$. No difference in the frequency of $\mathrm{CD} 4{ }^{+}$ $\mathrm{T}$ cells was observed between early cART patients and late cART patients. Although, a significant increase of these percentages was noted in early and late cART + patients, $\mathrm{CD} 4{ }^{+}$T-cell restoration remained incomplete as compared with seronegative individuals $(P<0.01)$ in both groups (Figure 1a). The frequency of $\mathrm{CD} 38^{+}$cells among $\mathrm{CD} 8^{+}$ $\mathrm{T}$ cells, a phenotypic marker of immune activation, was found significantly higher in the mucosa of naive cART HIV + patients $(60.4 \pm 9.9 \%)$ as compared with seronegative controls $(37.3 \pm 7.5 \% ; P<0.05)$. Interestingly, early cART patients displayed a significant lower frequency of $\mathrm{CD} 38^{+} \mathrm{CD} 8{ }^{+}$ $\mathrm{T}$ cells compared with late cART patients $(P<0.05)$ (Figure 1b).

Analysis of the epithelial barrier integrity was assessed by immunochemistry in rectal biopsies from HIV-1-infected patients and seronegative controls. As shown in Figure 1c for a representative analysis out of six patients from each group with similar findings, expression of the tight junction protein claudin-3 was found decreased in naive cART HIV + patients as compared with cART + and HIV seronegative individuals (Figure 1d).

\section{CART does not restore gut IL-17-secreting cells}

As IL-17 has an important role in gut homeostasis, we performed flow cytometry analysis of IL-17-producing lymphocytes following mitogenic stimulation of fresh isolated cells from rectal biopsies from HIV-1-infected patients and HIV seronegative donors (see Supplementary Figure S1 online). Several populations of IL-17-producing cells were phenotypically analyzed within $\mathrm{CD}^{+}{ }^{+}$(Th17), $\mathrm{CD} 8^{+}$T-cell populations (Tc17) and $\mathrm{CD}^{-}{ }^{-} \mathrm{CD} 8{ }^{+}$ILC-17. We also looked at other populations of mucosal $\mathrm{T}$ cells such as $\mathrm{CD}^{+}$ $\mathrm{CD} 4{ }^{-} \mathrm{TCR}-\gamma \delta^{+}$and MAIT cells $\left(\mathrm{CD} 3^{+} \mathrm{CD} 4^{-} \mathrm{TCR}-\gamma \delta^{-}\right.$ $\mathrm{CD} 161^{\mathrm{hi}} \mathrm{TCRV} \alpha 7.2^{+}$) described as capable to produce IL-17 in response to pathogens. ${ }^{21}$

Results showed a significant depletion of all subsets of IL-17producing lymphocytes in naive cART HIV + individuals as 


\begin{tabular}{|c|c|c|c|c|}
\hline Median (inter quartile range) or $\%$ & $\begin{array}{l}\text { Early cART } \\
\qquad(n=12)\end{array}$ & $\begin{array}{l}\text { Later cART } \\
\qquad(n=15)\end{array}$ & $\begin{array}{c}\text { Non-treated } \\
\quad(n=6)\end{array}$ & $\boldsymbol{P}$-value ${ }^{a}$ \\
\hline \multicolumn{5}{|l|}{ Demography } \\
\hline HIV risk group, \% & & & & 1 \\
\hline Heterosexual & $42 \%$ & $33 \%$ & $0 \%$ & \\
\hline Other & $0 \%$ & $0 \%$ & $17 \%$ & \\
\hline Ethnicity, \% & & & & 0.8 \\
\hline Caucasian & $92 \%$ & $80 \%$ & $100 \%$ & \\
\hline Sub-Saharan African & $8 \%$ & $20 \%$ & $0 \%$ & \\
\hline Year of HIV diagnosis & 2007 (2005-2008) & 1999 (1992-2003) & 2011 (2010-2012) & 0.0004 \\
\hline B & $0 \%$ & $33 \%$ & $0 \%$ & \\
\hline C & $8 \%$ & $7 \%$ & $0 \%$ & \\
\hline Co-infection, \% & & & & 1 \\
\hline None & $100 \%$ & $93 \%$ & $83 \%$ & \\
\hline HBV & $0 \%$ & $7 \%$ & $0 \%$ & \\
\hline $\mathrm{HCV}$ & $0 \%$ & $0 \%$ & $17 \%$ & \\
\hline \multicolumn{5}{|l|}{ Pre-ART } \\
\hline $\mathrm{CD}^{+}{ }^{+}$T-cell nadir, cells $\left.\mu\right|^{-1}$ & $522(380-640)$ & $205(110-303)$ & $436(195-582)$ & 0.0001 \\
\hline Age, years & 48 & 44 & 35 & 0.3 \\
\hline Duration with HIV, years & $4.8(4.0-6.3)$ & $13.2(8.2-19.8)$ & $0.5(0.1-2.4)$ & 0.0004 \\
\hline Overall cART exposure, years & $4.7(3.8-6.7)$ & $9.7(5.1-14.8)$ & NA & 0.02 \\
\hline Duration with $P V L<50$ copies $\mathrm{ml}^{-1}$, years & $4.2(3.5-5.9)$ & $7.5(4.6-11.3)$ & NA & 0.04 \\
\hline Current cART regimen, \% & & & NA & 0.2 \\
\hline NNRTI-based & $50 \%$ & $73 \%$ & & \\
\hline Pl-based & $50 \%$ & $20 \%$ & & \\
\hline II-based & $0 \%$ & $7 \%$ & & \\
\hline $\mathrm{CD}^{+}{ }^{+} \mathrm{T}$ cells $\mu \mathrm{I}^{-1}$ & $778(530-918)$ & $434(324-727)$ & $470(195-670)$ & 0.02 \\
\hline $\mathrm{CD}^{+}: \mathrm{CD}^{+}$ratio & $1.1(0.9-1.7)$ & $0.9(0.5-1.0)$ & $0.6(0.1-1.0)$ & 0.008 \\
\hline PVL, $\log _{10}$ copies $\mathrm{ml}^{-1}$ & $<1.3$ & $<1.3$ & $4.5(4.1-4.6)$ & 1.0 \\
\hline HIV-DNA level in blood, $\log _{10}$ copies per $10^{6}$ PBMCs & $1.8(1.6-2.4)$ & $2.6(2.4-2.9)$ & $3.0(2.9-3.2)$ & 0.0008 \\
\hline HIV-DNA level in gut, $\log _{10}$ copies per $10^{6}$ rectal cells & $1.7(1.3-2.1)$ & $2.1(1.8-2.5)$ & $2.9(2.4-3.0)$ & 0.056 \\
\hline
\end{tabular}

CART, combined antiretroviral therapy; CDC, Centers for Disease Control and Prevention; HBV/HCV, hepatitis B/C virus; Il, integrase inhibitors; MSM, men having sex with men; NA, not applicable or available; NNRTI, non-nucleoside reverse transcriptase inhibitors; PBMC, peripheral blood mononuclear cell; PHI, primary HIV infection; PI, protease inhibitors; PVL, plasma HIV-RNA viral load.

a Between the early cART and later cART groups. 

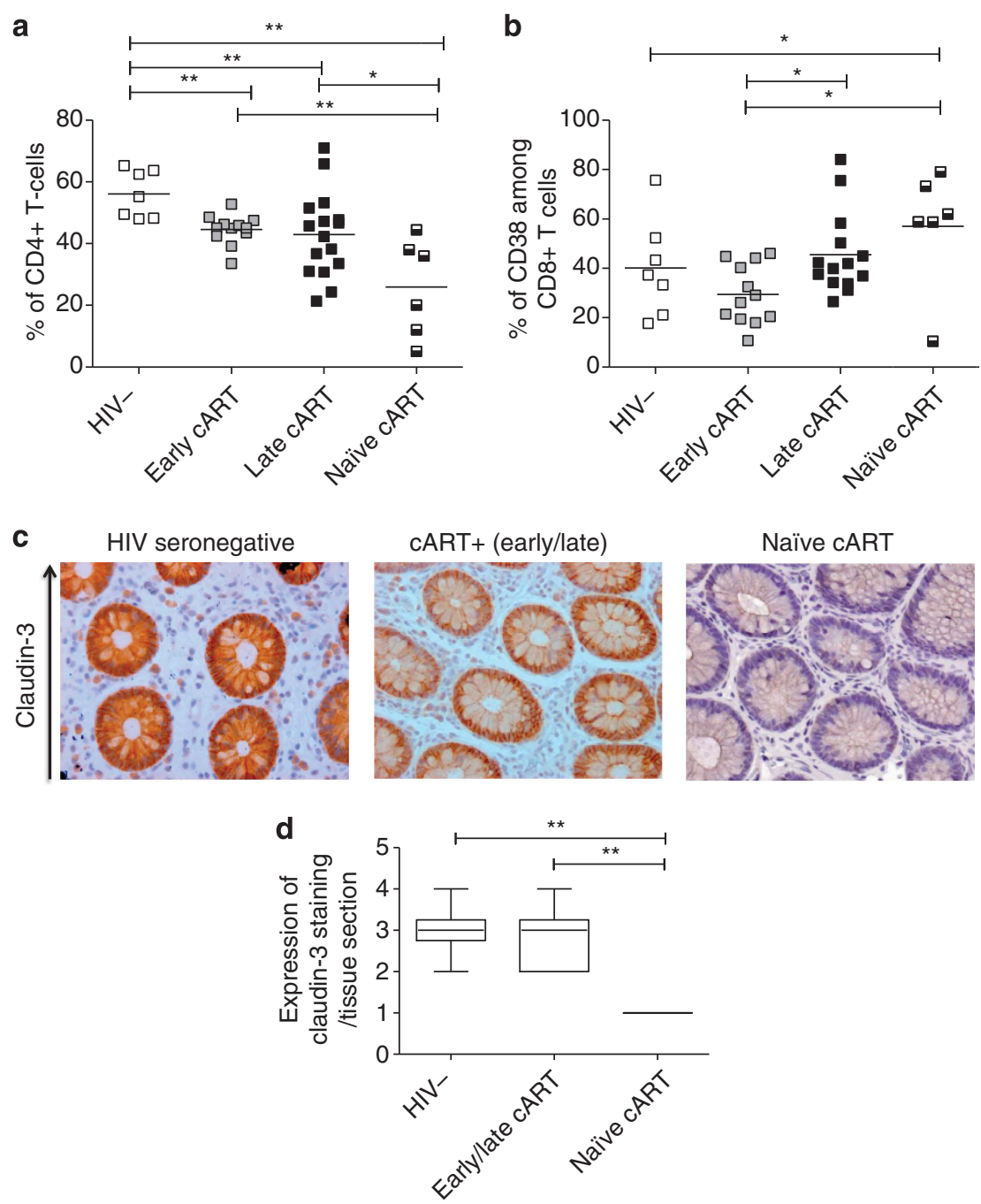

Figure 1 Mucosal CD4 ${ }^{+}$T cells are depleted in HIV-1-infected patients and are partially restored in combined antiretroviral therapy (cART) patients. The frequency of (a) $\mathrm{CD} 4^{+} \mathrm{T}$ cells and (b) $\mathrm{CD} 38^{+} \mathrm{CD} 8^{+} \mathrm{T}$ cells in the lamina propria lymphocytes of cART patients (early cART, gray boxes, $n=12$ and late cART, black boxes, $n=14$ ) or NT (naive cART HIV + patients, half filled boxes, $n=6$ ) or HIV seronegative patients (white boxes, $n=7$ ). (c) Representative image of Claudin-3 from healthy donor on the left panel, respective cART + patients on the middle, and cART - patients on the right panel. (d) Expression of Claudin-3-stained cells per tissue section in lamina propria lymphocytes of HIV seronegative, early/later cART, and naive cART. Statistical analyses were performed with nonparametric Mann and Whitney test. ${ }^{\star} P<0.05 ;{ }^{* \star} P<0.01$.

compared with HIV-1 seronegative controls. We observed depletion of IL-17-secreting T cells $(1.2 \pm 0.5 \%$ vs. $5.7 \pm 1.1 \%$; $P<0.01$; Figure 2a), Th17 cells $(5.0 \pm 1.4 \%$ vs. $10.2 \pm 1.8$; $P<0.01$; Figure $2 \mathbf{b})$, and ILC-17 cells $(1.1 \pm 0.3 \%$ vs. $3.0 \pm$ $0.6 \% ; P<0.05$; Figure 2d). These data were confirmed by a second immunohistochemical evaluation of the absolute numbers of $\mathrm{IL}-17^{+}$cells per unit area of lamina propria using paraffin-embedded rectal biopsies (data not shown). Consistent with previous report, ${ }^{5,22}$ globally the restoration of IL-17-secreting T cells remains incomplete in both groups of patients, the early treated and the lately treated, as compared with HIV-1 seronegative individuals ( $3.5 \pm 0.5 \%$ and $3.1 \pm 0.2 \%$ as compared with $5.7 \pm 1.1 \%$, respectively; $P<0.05$; Figure $2 \mathbf{a}$ ) while a substantial inter-individual heterogeneity of ILC-17 restoration in both groups was observed (Figure 2d).
Interestingly, naive cART HIV + patients displayed a significant lower frequency of Tc17 cells compared with late cART patients $(0.38 \pm 0.1 \%$ vs. $1.3 \pm 0.4 \% ; P<0.05$; Figure 2 c) .

\section{Lymphoid populations secreting IL-17 are altered in the mucosa of HIV-infected patients}

Additionally, we found that the frequency of $\gamma \delta \mathrm{T}$ cells were dramatically reduced in the rectal mucosa of HIV-infected patients naive of cART, or either early or late cART, as compared with HIV-1 seronegative controls (12.7 $\pm 6.0 \%$ and $17 \pm 7.0 \%$ and $16.2 \pm 3.08$ as compared with $35.0 \pm 5.4 \%$, respectively; $P<0.05$; Figure 3a). The frequency of MAIT cells varied among HIV-1-infected patients (Figure 3b). It tended to be higher in HIV-infected patients as compared with HIV-1 seronegative controls without reaching significance. 

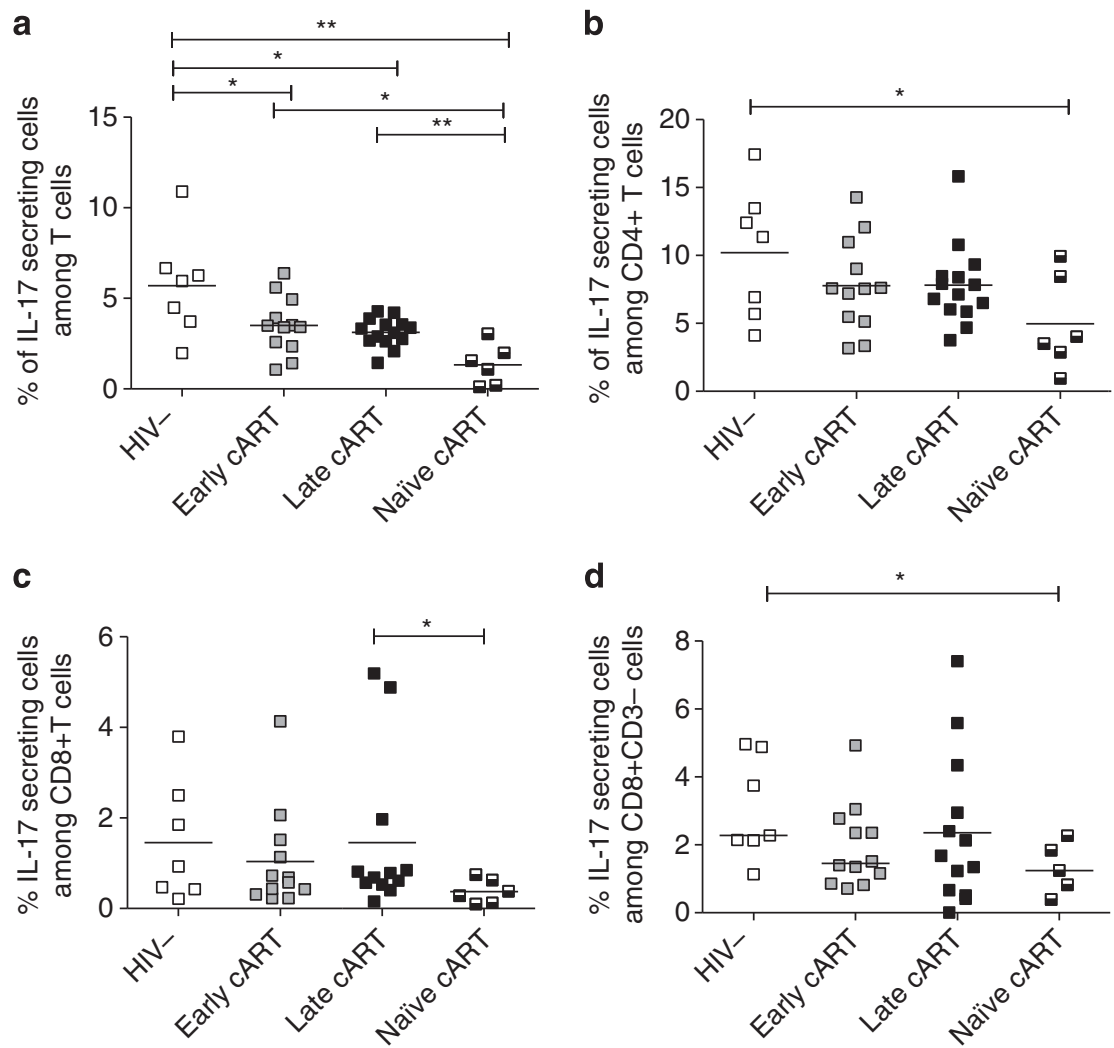

Figure 2 Interleukin (IL)-17-secreting cells are depleted during HIV infection and incompletely restored with the treatment. The frequency of IL-17secreting cells among (a) T cells, (b) CD4 ${ }^{+}$T cells, (c) CD8 ${ }^{+}$T cells, and (d) innate immune lymphocytes of combined antiretroviral therapy positive (cART + ) patients (early cART, gray boxes, $n=12$ and late cART, black boxes, $n=14$ ) or NT (naive cART HIV + patients, half filled boxes, $n=6$ ) or HIV seronegative patients (white boxes, $n=7$ ). Values are calculated as mean \pm s.e.m. Statistical analyses were performed with Mann and Whitney test. ${ }^{\star} P<0.05 ;{ }^{* *} P<0.01$.
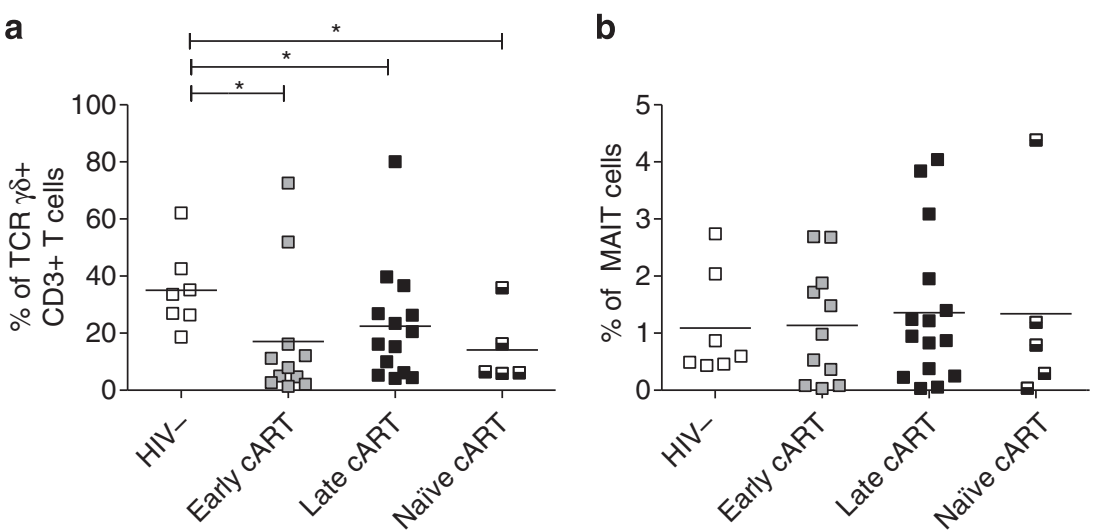

Figure 3 Lymphoid populations secreting interleukin-17 are altered in the gut mucosa of HIV-infected patients. The frequency of T-cell receptor (TCR)$\gamma \delta$ cells among (a) CD $3^{+}$intraepithelial lymphocytes and (b) mucosal-associated invariant T (MAIT) cells (CD $3^{+} \mathrm{CD}^{-}{ }^{-} \mathrm{TCR}^{-}{ }^{-} \delta^{-} \mathrm{CD} 161^{\text {hiTCRV }} \alpha 7.2^{+}$) among intraepithelial lymphocytes of combined antiretroviral therapy positive (cART + ) patients (early cART, gray boxes, $n=12$ and late cART, black boxes, $n=14$ ) or NT (naive cART HIV + patients, half filled boxes, $n=6$ ) or HIV seronegative patients (white boxes, $n=7$ ). Values are calculated as mean \pm s.e.m. Statistical analyses were performed with Mann and Whitney test. ${ }^{*} P<0.05$.

IL-22-producing $\mathrm{T}$ lymphocytes are preferentially restored in the gut of treated HIV patients.

Next, we looked at the effects of cART on cell populations producing IL-22 in fresh isolated cells from rectal biopsies from
HIV-1-infected patients and HIV-1 seronegative donors. We investigated changes of several IL-22-producing T-cell populations as defined as $\mathrm{Th} 22\left(\mathrm{CD} 3{ }^{+} \mathrm{CD} 4{ }^{+}\right), \mathrm{Tc} 22\left(\mathrm{CD} 3{ }^{+} \mathrm{CD} 8^{+}\right)$ and ILC-22 $\left(\mathrm{CD} 3^{-} \mathrm{CD} 8^{+}\right)$. We observed a depletion of 

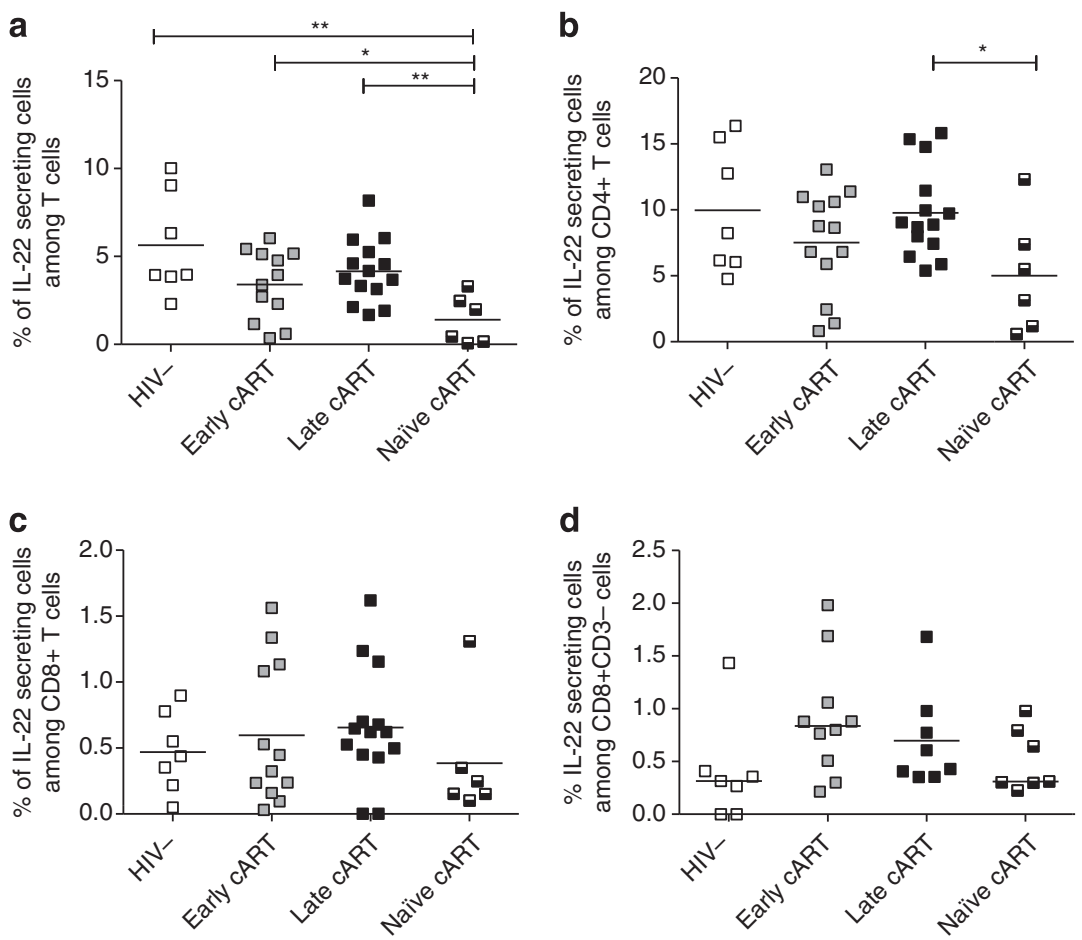

Figure 4 Reconstitution of interleukin (IL)-22-secreting cells during combined antiretroviral therapy (cART) treatment. The frequency of IL-22-secreting cells among (a) T cells, (b) CD4 ${ }^{+} \mathrm{T}$ cells, (c) CD8 ${ }^{+} \mathrm{T}$ cells, and (d) innate immune lymphocyte-22 in the lamina propria lymphocytes of cART + (early cART, gray boxes, $n=12$ and late cART, black boxes, $n=14$ ) or NT (naive cART HIV + patients, half filled boxes, $n=6$ ) or HIV seronegative patients (white boxes, $n=7$ ). Values are calculated as mean \pm s.e.m. Statistical analyses were performed with Mann and Whitney test. ${ }^{*} P<0.05$, ${ }^{* \star} P<0.01$.

IL-22-producing T cells in naive cART HIV + individuals as compared with HIV-1 seronegative controls $(1.4 \pm 0.6 \%$ vs. $5.6 \pm 1.1 \%$, respectively, $P<0.01)$ while in both groups of patients, the early treated and the late treated $(3.4 \pm 0.6$ and $4 \pm 0.5$ vs. $5.6 \pm 1.1 \%$, respectively, $P<0.05$ and $P<0.01$ ), the frequency of cells became no different from controls (Figure 4a). Precise phenotypic analysis suggests that the depletion of $\mathrm{T}$ cells producing IL-22 in naive cART HIV + patients is mainly driven by a loss of Th22 (5.0 $\pm 1.8 \%$; Figure $4 \mathbf{b}$ ) and CART treatment started during the early or late phase restored this population $(7.6 \pm 1.2$ and $9.8 \pm 0.9)$. In addition, the frequency of Tc22 cells among the different groups did not display any significant differences (Figure 4c). In contrast to ILC-17 cells, which were found to be depleted in naive cART HIV + individuals, no significant changes in ILC22 cells were observed among these patients $(0.5 \pm 0.2 \%$ vs. $0.4 \pm 0.2 \%$, respectively), while the frequency of this population tended to be higher in the early or late cART patients as compared with HIV seronegative controls $(0.8 \pm 0.2$ and $1.2 \pm 0.3$ vs. $0.4 \pm 0.2 \%$, respectively) (Figure $4 \mathbf{d}$ ).

FoxP ${ }^{+}$Treg are maintained in the gut during HIV infection Treg were defined as $\mathrm{CD} 4{ }^{+} \mathrm{CD} 25^{\text {high }} \mathrm{FoxP} 3^{+}$lymphocytes (see Supplementary Figure S1). We did not find significant differences in the frequency of these cells in freshly isolated cells from mucosal biopsies between HIV-1-infected patients and seronegative controls (see Supplementary
Figure S2A). These results were confirmed by immunohistochemical analysis in paraffin-embedded rectal biopsies showing that the absolute number of FoxP3 ${ }^{+}$cells did not differ significantly between naive, early and late cART + $\mathrm{HIV}+$ patients $(16.0 \pm 10$ and $13.0 \pm 4.0$ vs. $14.6 \pm 4.4$ FoxP3stained cells per unit area, respectively; see Supplementary Figure S2B,C).

\section{Viral load control is not sufficient to enable Treg/Th17 ratio restoration}

Recent reports from mice models, ${ }^{23}$ SIV pathogenic, and nonpathogenic models ${ }^{24}$ indicate that the balance between Th17 and Treg populations in the gut may better reflect the homeostatic response to inflammation and host defence against pathogens or commensal flora. ${ }^{23}$ Thus we determined for each group of HIV-1-infected patients and HIV seronegative donors the Treg/Th17 ratio in rectal mucosa. We found that naive cART HIV + patients exhibited a significantly higher Treg/ Th17 ratio as compared with HIV seronegative controls $(0.9 \pm 0.3 \%$ vs. $0.2 \pm 0.02 \%$, respectively, $P<0.01$; Figure 5a). Interestingly, this ratio tended to be lower in both groups of patients, the early treated and the late treated, but remained different from that observed in seronegative controls $(0.38 \pm$ 0.1 and $0.4 \pm 0.05$ vs. $0.2 \pm 0.02$, respectively; Figure 5a).

We found that the Treg/Th17 balance was inversely correlated with the frequency of $\mathrm{CD} 4{ }^{+} \mathrm{T}$ cells in the gut of HIV-1-infected patients $(r=-0.38, P=0.02)$ but not with 
a
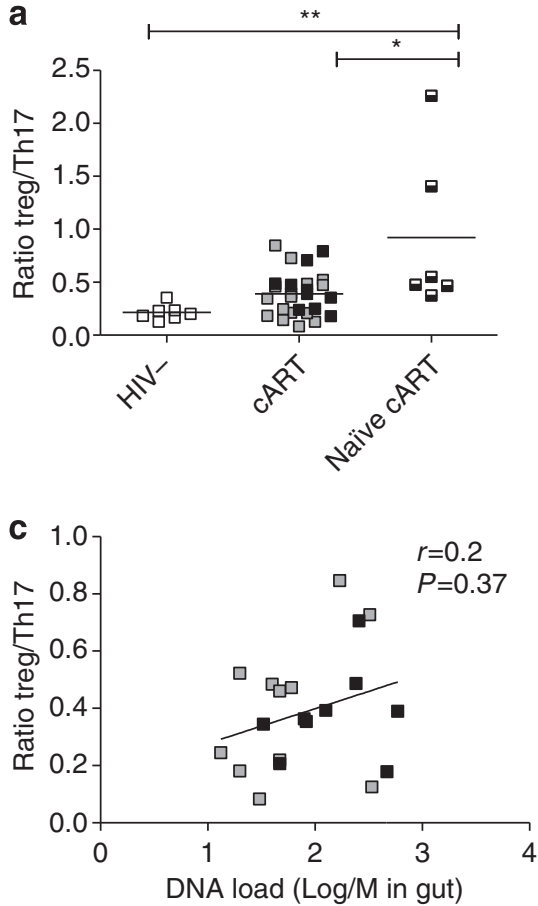

b
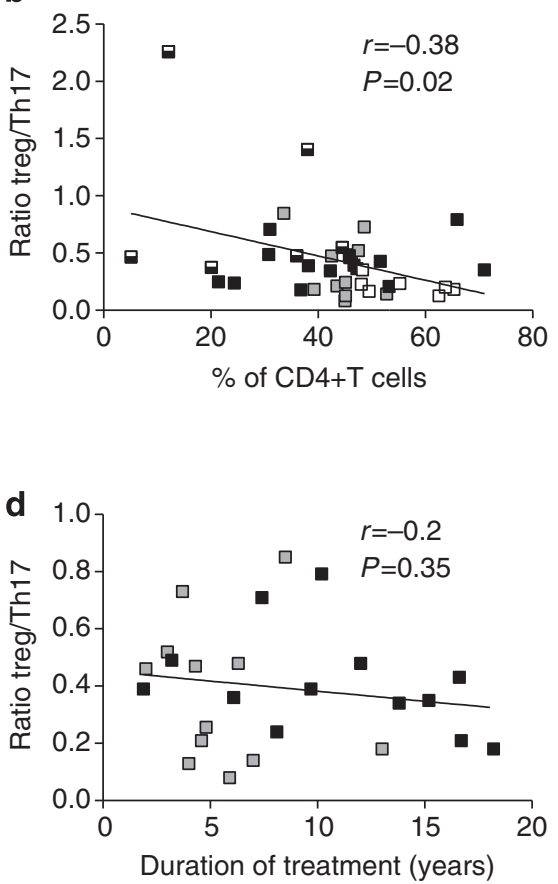

Figure 5 The regulatory T cells/T helper type 17 (Treg/Th17) balance inversely correlates with mucosal CD4 ${ }^{+}$T cells but not with DNA-HIV and treatment duration. (a) Treg/Th17 ratio.Correlation between ratio Treg/Th17 and (b) frequencies of mucosal CD4 ${ }^{+}$T cells, (c) DNA values in the gut (log/ $10^{6} \mathrm{cells}$ in gut) and in the lamina propria lymphocytes of combined antiretroviral therapy positive (cART + ) patients (early cART, gray boxes, $n=12$ and late cART, black boxes, $n=14$ ) or NT (naive cART HIV + patients, half filled boxes, $n=6$ ) or HIV seronegative patients (white boxes, $n=7$ ). (d) Correlation between Treg/Th17 ratio and treatment duration. Values are calculated as mean \pm s.e.m. Statistical analyses were performed with Mann and Whitney test and correlations using Spearman's rank order test. ${ }^{*} P<0.05 ;{ }^{* \star} P<0.01$.

the HIV reservoir $(r=0.2, P=0.37$ ) (Figure $5 \mathbf{c})$ as assessed by HIV DNA levels in gut mucosal. We looked at the interaction between Treg/Th17 balance and markers of systemic immune activation. There were no clear correlations between Treg/ Th17 ratio and blood $\mathrm{CD} 38^{+} \mathrm{DR}^{+} \mathrm{CD} 4{ }^{+}$frequency $(r=0.32$, $P=0.07), \mathrm{CD} 38^{+} \mathrm{DR}^{+} \mathrm{CD} 8^{+}$frequency $(r=0.26, P=0.14)$, and plasma IP10 $(r=0.4, P=0.06)$ in HIV-infected patients (data not shown) and treatment duration (Figure 5d). We observed that $50 \%$ of early treated patients reached a ratio close to seronegative controls as compared with $30 \%$ of later treated patients despite a lower duration of cART (4.7 vs. 9.7 years, respectively; $P=0.02$ ).

\section{The timing of CART initiation discriminates two profiles of gene expression in the gut of HIV + patients}

Next, we performed gene arrays analysis on mRNA isolated from whole blood and gut biopsies from cART + HIV + patients. Unsupervised clustering analysis of whole-blood gene expression did not show significant differences between patients (data not shown). However, analysis of gut biopsies led to the clear identification of two clusters (Figure 6a), with 1572 genes differentially expressed (adjusted for age and after FDR (false discovery rate) control $<0.05$ ). Interestingly, cluster 1 comprised a majority of later treated patients $(8 / 11)$ while early treated patients belong in majority in cluster 2 (8/11). As the timing of treatment initiation was associated with the duration of treatment and the duration of
HIV infection in this observational study, we performed a multivariable analysis to look at the independent contribution of these three factors. In this multivariable logistic model, the only one factor associated with classification in cluster 1 compared with cluster 2 was the timing of cART initiation $(P=0.05)$. The duration of HIV and the duration of cART exposure were not significantly associated with the clusters $(P=0.85$ and 0.94 , respectively).

Furthermore, supervised gene expression analysis in the blood of these two clusters confirmed the lack of distinct expression profiles (data not shown). Our results demonstrate the absence of correlation between blood and the gut in the profile of gene expression and emphasize the importance of gut mucosal homeostasis.

\section{Early cART initiation prevents damage of epithelial barrier and intestinal lymphoid structure}

Analysis of key differentially regulated genes demonstrated the induction of genes strongly associated with the maintenance of gut integrity (Figure 6b). First, canonical pathway showed a variation between clusters in epithelial adherence junction signal (Figure 6d). Hence, reduced expression levels of genes such as E-cadherin and nectin, which are known as cell-cell adhesion molecules, were found in the cluster 1 enriched in later treated patients (late cART). Moreover, IL-22, S100A8 (antimicrobial peptides) and CCR6 genes were increased in the cluster 2 enriched in early treated patients (early cART) 
compared with cluster 1 (late cART) (Figure 6c), suggesting that epithelium integrity is maintained in early treated patients. Second, upregulation of genes of dendritic cell (DC) maturation, including CD40, CD83, CD86, CCR7 and major histocompatibility complex class II, were observed in cluster 2 (early cART) compared with cluster 1 (late cART) (Figure 6b-e). IL-6 and IL12p40 transcripts, two innate cytokines secreted by DC and involved in the differentiation of Th17 cells were increased in early cluster (Figure 6c). Third, genes involved in metabolism pathway are decreased in cluster 2 (early cART) compared with cluster 1 (late cART), suggesting profound perturbations in metabolism and adsorption (Figure 6b). Other genes involved in hematological and system development and functions, including activation of immune cells mobility of leukocytes, were differentially expressed between the two clusters (Figure 6b, see Supplementary Figure S3). We also show that the increased expression of tumor necrosis factor- $\alpha$ represented a significant node within the network connecting lymphotoxin- $\beta$, CR2, and CD19 genes, all known to be important in the maintenance of lymphoid structure. Moreover, the pathway related to the increased expression of tumor necrosis factor gene in cluster 2 (early
cART) connects genes involved in the integrity of DC function (Figure 7a).

Finally, we found that genes characterizing follicular DCs (FDCSP-CR2) are increased in cluster 2 (early cART) compared with cluster 1 (late cART) (Figure 6b) together with an increase of lymphotoxin- $\beta$ and IL-7 receptor. This, and the canonical pathway shown in Figure 7a, suggests that the integrity of lymphoid tissue (LT) structure is maintained in early treated patients.

To confirm these data, we performed immunohistochemistry on rectal biopsies from every treated patient included in this study. Because of the size of the biopsies, we could analyze six early treated patients and seven late treated patients. We found preservation of secondary follicles with germinal center in early treated patients associated with a striking network of CD $21^{+}$follicular DCs (FDC) (Figure 7b). By contrast, most of the later treated patients displayed limited lymphoid infiltrates or primary follicles lacking germinal center formations and $\mathrm{CD} 21^{+}$FDC network (Figure 7c). We defined isolated lymphoid follicles as appropriate CD3 network around germinal center with a striking network of $\mathrm{CD} 21^{+}$FDC. As shown in Figure $\mathbf{7 d}$, five out of the six early cART patients

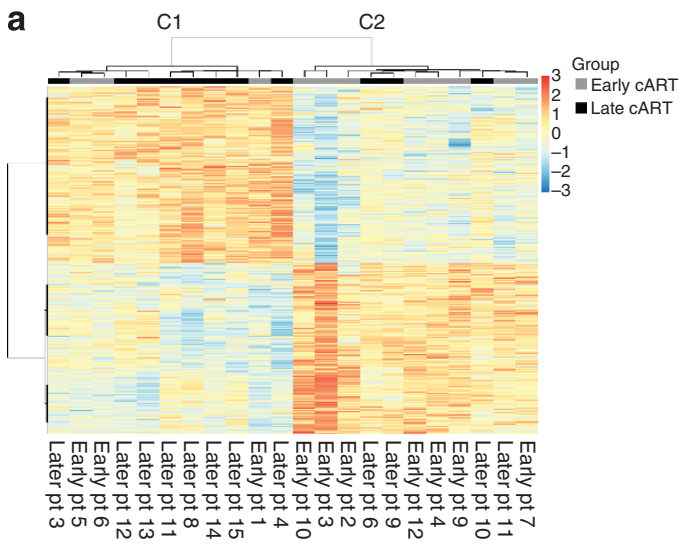

b
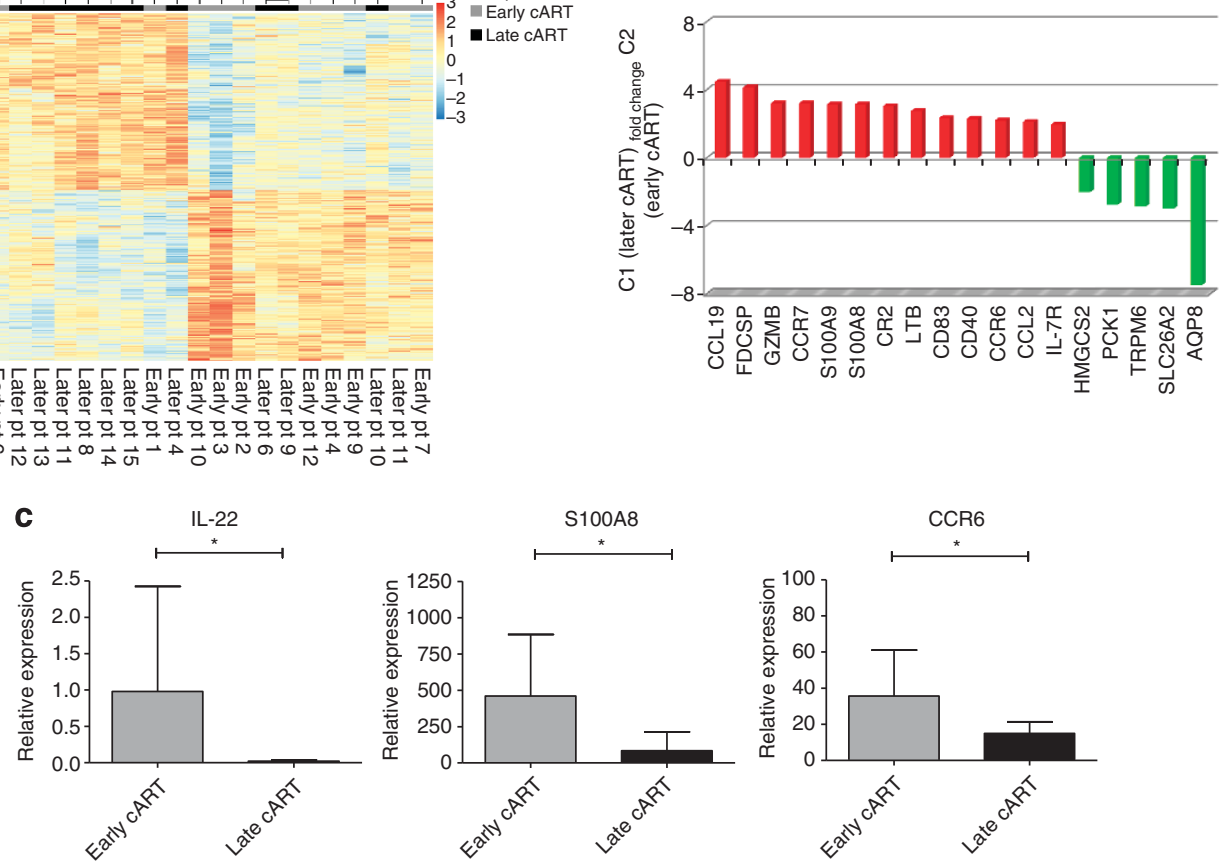

Figure 6 Transcriptomic analysis-identified upregulated genes essential for mucosal homeostasis in early combined antiretroviral therapy (cART) patients. (a) Identification of two clusters using unsupervised clustering (ward method and Euclidean distance). Cluster 1 comprises a majority of later treated patients (8/11) while early treated patients belong in majority in cluster 2 (8/11). Gene expression was row scaled. (b) Fold change of selected genes that were upregulated in early cluster (top) and later cluster (bottom). (c) Interleukin (IL)-22, S100A8, IL-6, and C-C motif chemokine receptor 6 (CCR6)-specific mRNA expressions (by quantitative PCR) on the gut biopsies of treated patients (Cluster 1 (later cART), $n=11$ and Cluster 2 (early CART), $n=11){ }^{*} P<0.05$. (d) Canonical pathway from Ingenuity Pathway Analysis (IPA) in epithelial adherence junction signal. Red indicates upregulation (fold change $>1.5$ ), and green indicates downregulation in cluster 1 (fold change $<1.5$ ). (e) The pathway involved in dendritic cell maturation from IPA analysis for selected genes is shown. Red indicates upregulation (fold change $>=1.5$ ); green indicates downregulation (fold change $<=-1.5$ ); grey indicates genes whose expression values are between -1.5 and 1.5. Shape: triangle represents kinase; square represents cytokine; rectangle represents ligand-dependent nuclear receptor; diamond represents enzyme; trapezoid represents transporter; ellipse represents transcription regulator; and circle represents others. 
d

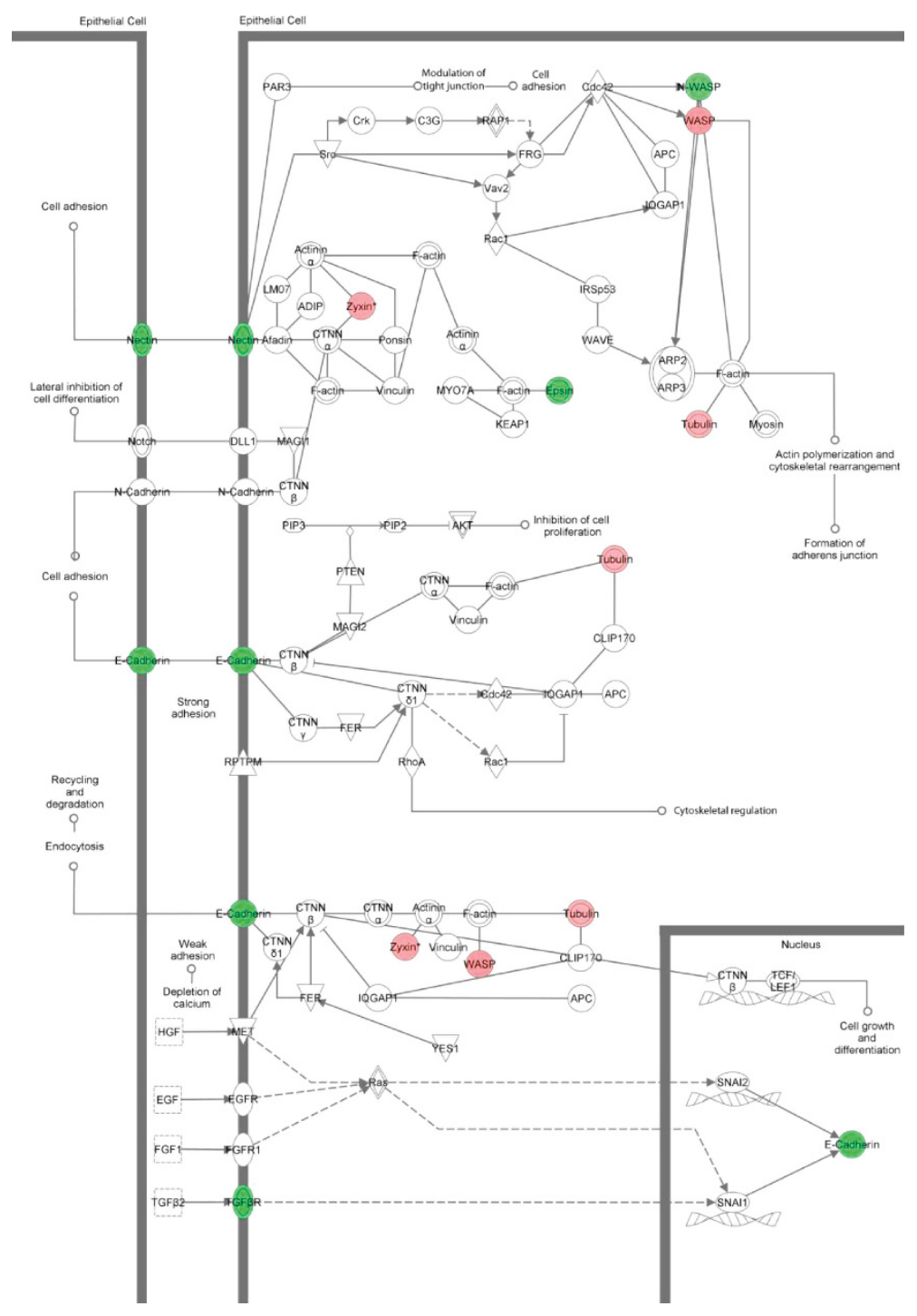

e

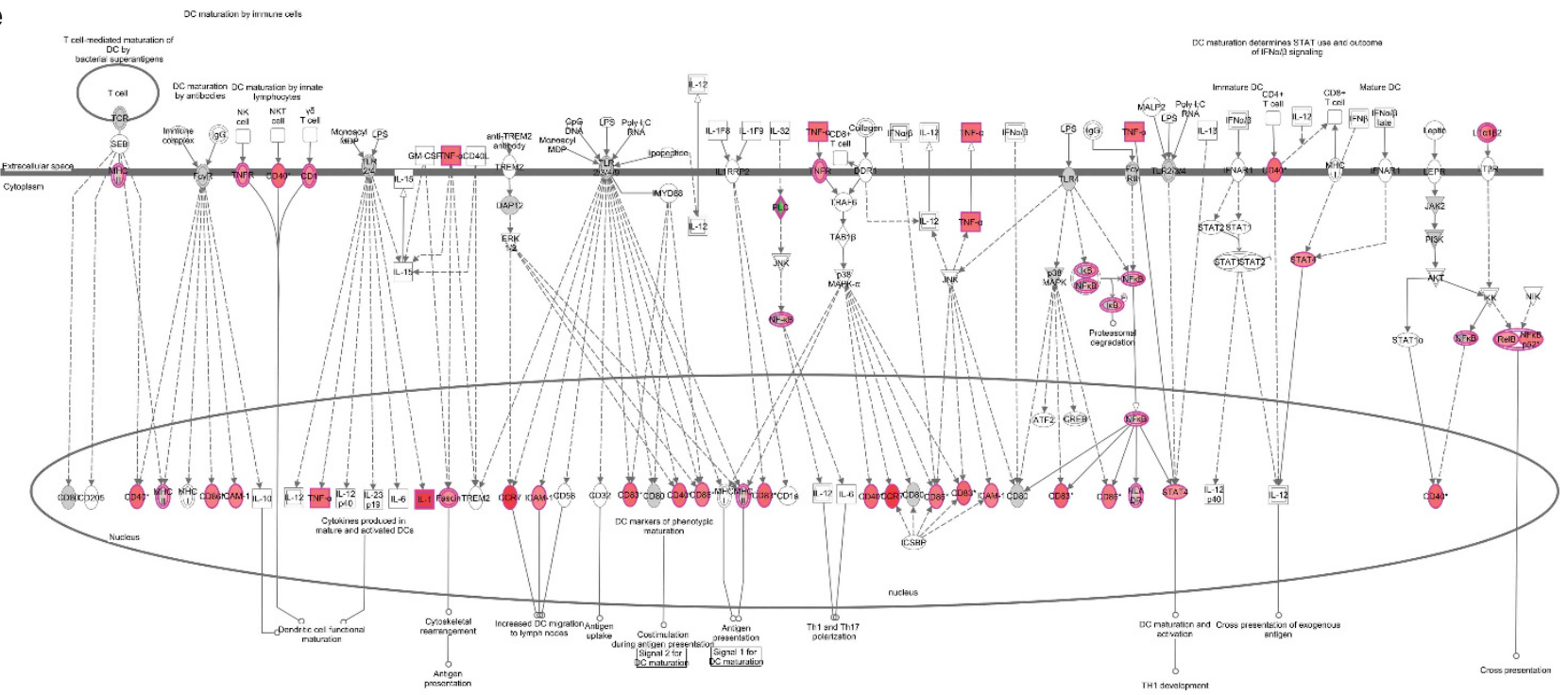

Figure 6 Continued. 


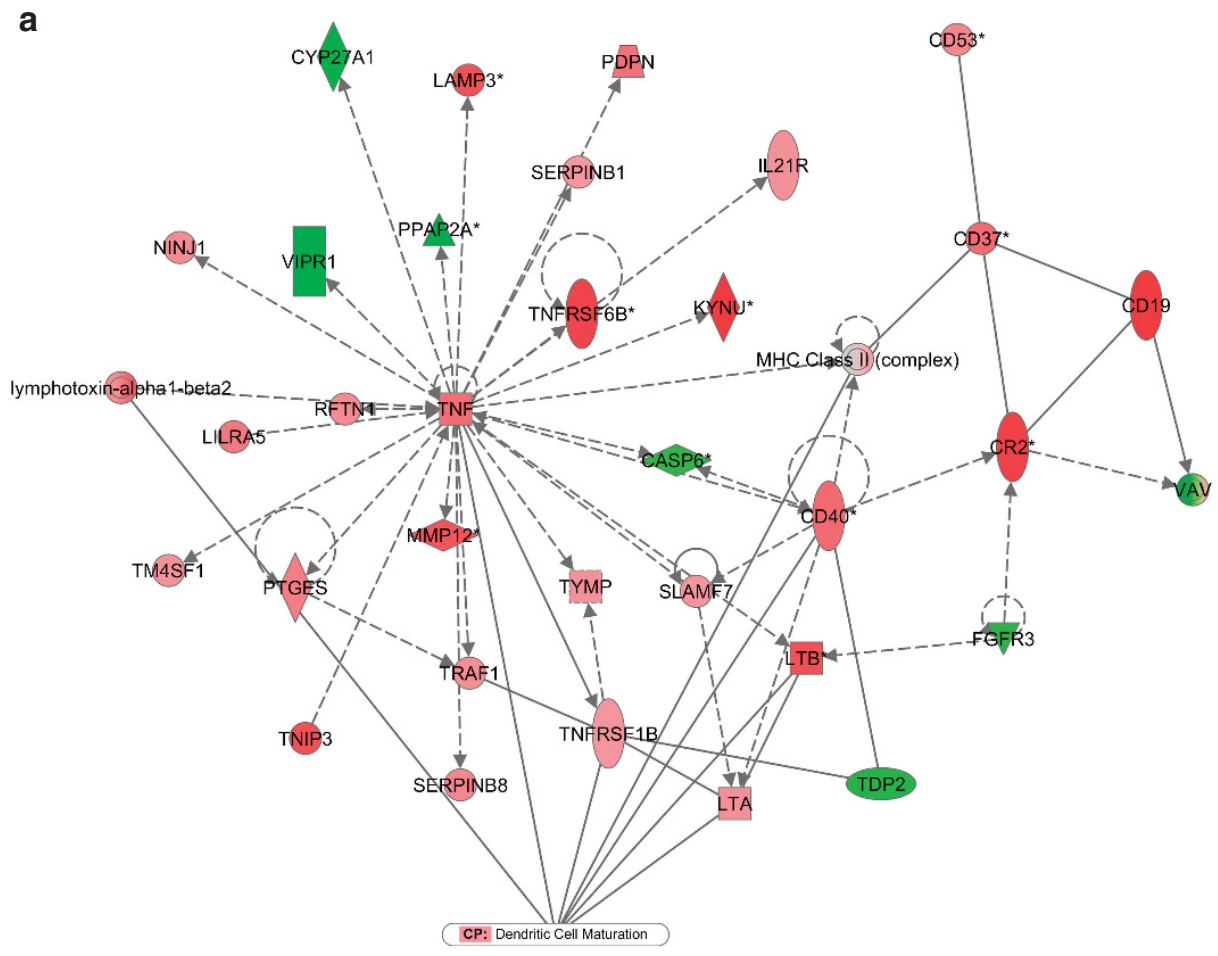

b

Early cART

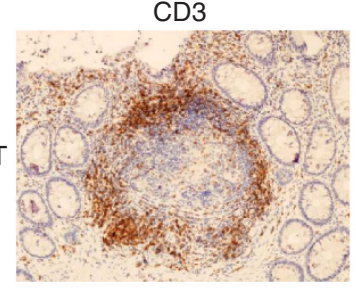

C

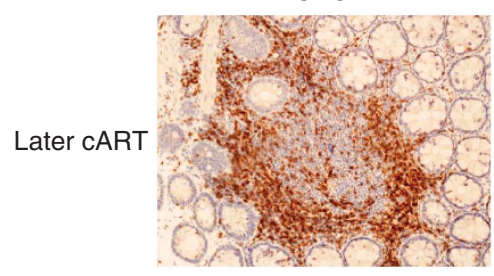

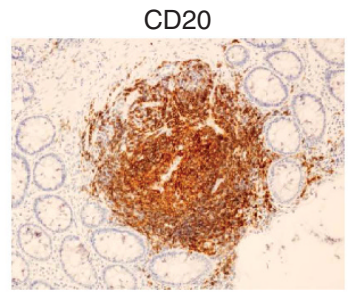

CD20

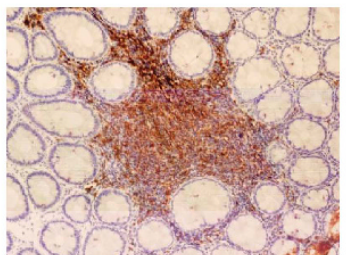

CD21

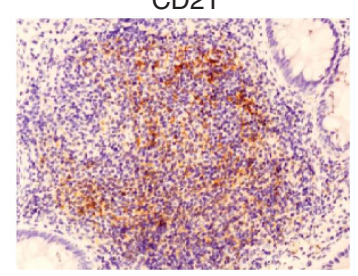

CD21

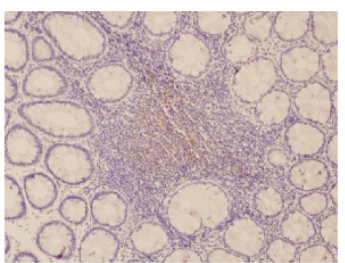

d

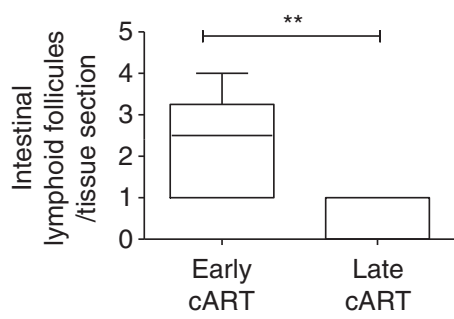

\begin{tabular}{|l|c|c|}
\cline { 2 - 3 } \multicolumn{1}{c|}{} & $\begin{array}{c}\text { Early } \\
\text { cART }\end{array}$ & $\begin{array}{c}\text { Late } \\
\text { cART }\end{array}$ \\
\hline & $n=6$ & $n=7$ \\
\hline$\geq 2$ ILF & 5 & 2 \\
\hline$\leq 2$ ILF & 1 & 5 \\
\hline
\end{tabular}

Figure 7 The gut lymphoid structure is preserved in early combined antiretroviral therapy (cART)-treated patients. (a) The network involved in lymphoid tissue structure from Ingenuity Pathway Analysis for selected genes is shown. Red indicates upregulation (fold change $\geqslant 1.5$ ); green indicates downregulation (fold change $\leqslant-1.5$ ); grey indicates genes statistically significant and whose expression values are between -1.5 and 1.5 .

Shape: triangle represents kinase; square represents cytokine; rectangle represents ligand-dependent nuclear receptor; diamond represents enzyme; trapezoid represents transporter; ellipse represents transcription regulator; and circle represents others. Representative image of CD3, CD20, and CD21 from (b) early treated patients showing a well-organized B-cell follicle containing a germinal center highlighted by a network of CD21 ${ }^{+}$FDC and (c) later treated patients with a small primary B-cell follicle without CG and few FDC. (d) The number of lymphoid follicles per tissue section and table showing the numbers of intestinal lymphoid follicles (ILF) assessed by immunohistochemistry in rectal biopsy of early vs. late cART patients. Values are calculated as mean \pm s.e.m. Statistical analyses were performed with Mann and Whitney test. ${ }^{\star *} P<0.01$. 
displayed more than two intestinal lymphoid follicles, whereas only two out of the seven late cART patients displayed it. These results suggest that the timing of cART initiation may preserve or restore LT structures in the gut of HIV-1-infected patients.

Altogether, these results demonstrated that early treated patients displayed better key functions involved in the maintenance of gut integrity and cell homeostasis.

\section{DISCUSSION}

In this study, we show that $\mathrm{CD} 4^{+}$and $\mathrm{CD} 8^{+} \mathrm{T}$ cells and $\mathrm{CD} 3^{-} \mathrm{CD} 8{ }^{+}$lymphocytes producing $\mathrm{IL}-17$ or $\mathrm{IL}-22$ are significantly decreased in the gut mucosal of HIV-infected individuals. Although the defect in IL-17-secreting cells persists despite long-term control of viral load, we show that IL-22secreting cells are significantly restored in patients treated with cART. We also show that the ratio between Treg and Th17 cells in the mucosa reflects better the disturbance of gut homeostasis in HIV infection. Our results point out that early initiation of cART may predict restoration of a Treg/Th17 ratio close to non-HIV-infected controls independently of the gut reservoir and peripheral $\mathrm{CD} 4 \mathrm{~T}$-cell counts. This phenotypic analysis was consistent with the results of the transcriptomic analysis performed in the same mucosal biopsies. We show an increased expression of genes important for enterocyte integrity and mucosal immune function in the gut mucosal from a majority of individuals treated at the early phase of the infection as compared with patients treated later. Moreover, we show that patients treated at the chronic phase exhibited consistently an increased expression of genes involved in mucosal metabolism. Our results contribute to demonstrate that early treatment initiation has an essential role in maintenance of the gut homeostasis.

As described by others, we found that despite several years of cART reconstitution of gut $\mathrm{CD} 4{ }^{+} \mathrm{T}$ cells and Th17 remains typically incomplete ${ }^{25-27}$ as compared with HIV - controls. Of note, we cannot rule out that in our study as in other papers from the literature ${ }^{28,29}$ the selection of HIV seronegative control undergoing colonic surgery might represent a bias. We now provided evidence that all lymphocytic sources of IL-17 are also depleted in the mucosa of HIV-infected individuals. We show that the frequencies of $\gamma \delta$ T cells and ILC-17, but not MAIT cells, are decreased in HIV infection. Several experimental models have shown that $\gamma \delta \mathrm{T}$ cells may potentiate or inhibit Th17 and Treg cell functions, respectively ${ }^{30,31}$ suggesting that the loss of ILC-17 and $\gamma \delta$ T cells may also contribute to the impairment of intestinal integrity in HIV infection. Albeit decreased in the periheral blood of HIV-infected patients, ${ }^{32}$ we cannot rule out that the normal frequency of MAIT cells results from a homing of these cells in the gut in response to viral replication as described in other infection. ${ }^{33}$ We show that, in contrast to Th17, the frequency of IL-22-secreting T cells is restored to a normal range in patients treated with cART as compared with naive individuals. We hypothesize that this population belongs to a newly described subset of Th22 cells that produces IL-22 independently of interferon- $\gamma$ and IL-17. ${ }^{34,35}$ Interestingly, experimental models have shown that these cells may be involved in tissue reparation. ${ }^{36}$ The role of these cells in HIV infection requires further studies, but their recruitment in the gut may participate to maintain barrier integrity in the context of $\mathrm{CD} 4^{+}$T-cell depletion.

Several studies have described an imbalance of specific cell populations in the gut of HIV/SIV-infected individuals. However, one level of complexity is represented by the interaction and the crosstalk between these populations. ${ }^{37}$ An overlapping developmental diversity between Treg, Th17, and Th22 modulated by environmental cues, such as cytokines and metabolic factors, has been described. We show here that even treated in the long term patients who started cART at the chronic phase of the infection experienced an increase expression of metabolic genes which could impact locally function and distribution of mucosal lymphoid populations. Thus, despite a profound CD4 depletion, Tregs were maintained in the gut of patients, suggesting a process of active recruitment into and/or expansion within the intestinal mucosa in response to local inflammation.

On the other hand, suppression of HIV-specific T-cell responses by Tregs may also be harmful to the host, because this may contribute to the anergy of the immune system to HIV. ${ }^{38}$ Given the reciprocal relationship between Th17 and Treg, it has been suggested that the balance of Treg and Th17 may, in fact, be more important than the absolute levels of either one independently. ${ }^{24}$ Loss of Th17 to Treg balance during acute infection is related to and predictive of SIV disease progression, and a normal Treg/Th17 ratio was associated with limited immune activation and no disease progression. A recent study has shown that the Treg/Th17 ratio in the blood of acutely infected patients is correlated to the viral set point. ${ }^{39}$ Interestingly, our data extend significantly those studies showing that the timing of cART initiation in HIV may have a role in the restoration of gut Treg/Th17.

Noteworthy, the unsupervised analysis of gene expression in gut biopsies from 22 patients discriminated two groups of patients according to the timing of cART initiation. Analysis of key differentially regulated genes demonstrated that at least three important pathways are expressed differentially between patients treated early or later. First, early patients display a significant induction of genes that are strongly associated with the maintenance of epithelium integrity. These data confirmed the persistence of intestinal epithelial damage, caused by loss of intestinal epithelial cells and disruption of tight junctions even in patients with a significant restoration of $\mathrm{CD} 4{ }^{+} \mathrm{T}$-cell counts at the periphery after several years of successful cART. Second, and consistently, early treatment maintains features of DC maturation in gut mucosal, which suggest the capacity of GALT of these patients to mount an adapted immune response. By contrast, we may suggest that the impairment of DC function in HIV-infected patients hampers the capacity of mucosal DC subsets to deliver signals necessary for the differentiation of IL-17-producing cells. ${ }^{6}$

Finally, the data reported herein show clearly that early cART maintains LT structures. This observation may reconcile the lack of restoration of gut Th17 cells and a better control of 
microbial translocation and a lower systemic activation in early treated patients. Indeed, experimental models have shown that these structures may exert a compensatory mechanism to contain the intestinal microbiota in the absence of Th17. ${ }^{40}$ Moreover, the T-cell zones of these LT structures provide survival signals to naive $\mathrm{CD} 4{ }^{+} \mathrm{T}$ cells, such as IL-7 and selfantigen-major histocompatibility complexes. Recent studies have shown that the loss of fibroblastic reticular cell (FRC) network and collagen deposition in pathogenic HIV/SIV infections leads to the lack of IL-7 production limiting the survival of naive $\mathrm{T}$ cells. Consistently, using transcriptomic and HIC approaches, we show a balance between IL-7 and preservation of FRC in early patients, which contrasts with a decreased production of lymphotoxin- $\beta$ and a loss of FRC networks in late treated patients. ${ }^{41}$

One another lesson from this study is that transcriptomic analyses in the blood did not show any differences between patients whatever the time of cART initiation, pointing out the importance of restoration of gut homeostasis during HIV infection. Gut transcriptomic analysis was performed on biopsy isolated from the rectum. However, it would be interesting to analyze other gut mucosal sites that differ from rectum in terms of density of commensal flora and Peyer's patches. Globally, these results highlight that some patients displayed features important to limit immune activation and to improve immune reconstitution. We provide evidence that the primary determinant of gut homeostasis restoration is the timing of cART initiation. We believe that by limiting inflammation driven by the viral replication, gut homeostasis can be partially restored and/or protected as in nonpathogenic models of lentiviral infection. However, we cannot exclude that other factors, such as alteration in the composition of the commensal flora, participate to the dysbalance between Th17 and Treg in the gut mucosal in HIV-infected patients as recently shown in pathogenic models of SIV infection. ${ }^{23}$

Our study raises concerns as to how to restore/maintain gut homeostasis during HIV infection. We show here the large immunological benefit of initiating treatment at the early phase of the infection. Interestingly, one of us (L.H.) has reported a cohort of $14 \mathrm{HIV}$ patients post-treatment controllers (PTCs) whose viremia remained controlled for several years after the interruption of prolonged cART initiated during the primary infection have been identified. Most PTCs lacked the protective HLA-B alleles that are over-represented in spontaneous HIV controllers. Accordingly, the PTCs had poorer $\mathrm{CD} 8{ }^{+} \mathrm{T}$-cell responses, and long-lived HIV-infected $\mathrm{CD}^{+}{ }^{+} \mathrm{T}$ cells contributed poorly to the total resting HIV reservoir in the PTCs. ${ }^{19}$ The main question is why PTCs are capable to control the infection after therapy interruption. Likely, regarding the limited number of these patients among those who have initiated cART at the primary phase of the infection, early cART is one, but not the exclusive, condition that may explain this condition. Therefore, to characterize host conditions could help to answer to this question. We have performed flow cytometry analysis and transcriptomic analysis on rectal biopsy of two PTCs not included in the cohort analysis reported here.
Interestingly, characteristics of these patients fit completely with early cART patients exhibiting a Treg/Th17 ratio $<0.3$ and a gene expression profile similar to that of the cohort of early cART (data not shown).

From a clinical stand point, symbiotic probiotics supplementation of cART may enhance gastrointestinal immune function and increased reconstitution of colonic $\mathrm{CD} 4^{+} \mathrm{T}$ cells, possibly by increasing antigen- presenting cell frequency and/ or function and decreasing inflammation-associated fibrosis. ${ }^{42}$ Cytokine therapy with IL-7 leads to a peripheral ${ }^{43}$ improvement of T-cell counts but also improves gut homeostasis through induction of T-cell expression of integrin $\alpha 4 \beta 7$ promoting naive $\mathrm{T}$-cell homing to the intestinal mucosa. ${ }^{44}$ We have reported that IL-7 exerts in vitro and in vivo in IL-7treated HIV-infected patients two synergistic effects on Treg cells by modulating CD39 and increasing ATP receptor leading to a switch of Treg cells towards a Th17 phenotype. ${ }^{43}$ Thus further studies are needed to explore ways to accelerate the reconstitution of GALT homeostasis in the majority of patients who have been initiated cART at the chronic phase of the infection.

In conclusion, we provide here a strong rationale for initiating cART as soon as possible during the acute phase of HIV infection in order to accelerate and/or protect gut homeostasis from local damages.

\section{METHODS}

Ethics statement. All of the subjects provided their informed written consent to participate in the study. This study was funded by ANRS sponsored by Orléans Regional Hospital and approved by the Tours ethics review committee.

Cells isolation. Rectal biopsies were collected by rectoscopy at the "Centre Hospitalier Regional d'Orléans". To obtain intraepithelial lymphocytes, cells subjected to two rounds of vigorous shaking RPMI medium supplemented with $1 \%$ fetal bovine serum, $1 \mathrm{~mm}$ EGTA, and $1 \mathrm{M} \mathrm{MgCl}_{2}$. To obtain lamina propria lymphocytes (LPL), additional two rounds of tissue digestion in medium containing collagenase II $100 \mathrm{U} \mathrm{ml}^{-1}$ (Sigma-Aldrich, Saint-Quentin Fallavier, France) followed by mechanical disruption with a syringe equipped with an 16-gauge blunt-end needle were performed. Both intraepithelial lymphocytes and LPL were pooled and washed in R10 medium (RPMI 1640 (Bio Whittaker Europe, Verviers, France) supplemented with $10 \%$ fetal bovine serum (Gibco, Paisley, United Kingdom), $1 \%$ penicillin and $1 \%$ streptomycin (Invitrogen, Carlsbad, CA). Cells were destined for immunophenotyping directly.

Cell culture and immunophenotypic analyses. To study the cytokine secretion, isolated rectal LPL were stimulated with phorbol 12 -myristate 13 -acetate $\left(100 \mathrm{ng} \mathrm{ml}^{-1}\right)$ and ionomycin $\left(1 \mu \mathrm{g} \mathrm{ml}^{-1}\right)$ in the presence of a protein transport inhibitor, Brefeldin A, while to study Tregs, rectal LPL were stimulated with in R10 media with Brefeldin $\mathrm{A}\left(10 \mu \mathrm{g} \mathrm{ml}^{-1}\right)$ alone for $5 \mathrm{~h}$ at $37^{\circ} \mathrm{C}$ and $5 \% \mathrm{CO}_{2}$. Cells were stained with CD3 A700 (UCHT1, BD Biosciences, Le Pont de Claix, France), CD4 PECF594 (RPA-T4, BD Biosciences), CD8 APC H7 (SK1, BD Biosciences), CD25 APC (2A3, BD Biosciences), and CD39 PE (TÛ66, BD Biosciences). In order to exclude dead cells from the analysis, the LIVE/DEAD fixable dead cell stain kit for $405 \mathrm{~nm}$ excitation (Molecular Probes, Saint Aubin, France; Invitrogen) was used in all stainings. Cells were then fixed with Stain Buffer (BD Biosciences) and permeabilized using the Human FoxP3 Buffer Set 
(BD Biosciences). For intracellular staining, cells were incubated with IL-17 PerCp-Cy 5.5 (eBio64DEC17, eBioscience, San Diego, CA), IL-22 PC7 (22URTI, eBioscience), and FoxP3 V450 (259/D7, eBioscience). Following a lymphocyte gate and dead cell discrimination, live cells were further gated for $\mathrm{CD}^{+}{ }^{+} \mathrm{T}$ cells. $\mathrm{CD} 3^{+}$ $\mathrm{T}$ cells were then plotted as $\mathrm{CD} 4^{+}$vs. $\mathrm{CD} 8{ }^{+} \mathrm{T}$ cells. The secretion of IL-17 and IL-22 were studied in each T-cell subset after 5-h mitogenic stimulation while Treg expression was studied among nonstimulated $\mathrm{CD}^{+}{ }^{+}$T-cell subset (defined as CD25 $5^{\text {high }}$ and FoxP3 ${ }^{+}$). Cytometry acquisition was performed on LSR II (BD Biosciences), and data were analyzed using the Flowjo (Version 7.6.5; TreeStar, Ashland, OR) software.

Immunohistochemistry. Deparaffinized tissue sections were stained for CD20, CD3 (DakoCytomation, Glostrup, Denmark) and CD21 (Novocastra, Newcastle, UK) using a three-step immunoperoxidase technique on a BOND-III Autostainer (Leica Microsystems, Newcastle-upon-Tyne, UK) according to a protocol described elsewhere. $^{45,46}$ For Claudin-3 (1:100 dilution, Thermo Scientific, Fontenay-sous-Bois Cedex, France) and FoxP3 (1:50 dilution, Abcam, Cambridge, UK), a manual technique with an avidin-biotin complex system (ABC kit, Vectastain, Les Ulis Cedex B, France) was applied after appropriate antigen retrieval with citrate/EDTA (pH6) buffer. The staining of the cells was performed using the DAB Substrate Kit for peroxidase (Vector Laboratories, Les Ulis Cedex B, France) or Red Alkaline Phosphatase Substrate Kit I (Vector Laboratories). All slides were counterstained with hematoxylin. Immuno-histochemical images were acquired on a Zeiss Axioplan 2 (Göttingen, Germany) microscope equipped with $\times 20(0.45$ NA) objective, using a Zeiss Mrc digital camera (Göttingen, Germany) and axiovision program (Göttingen, Germany).

Microarray analysis. Gene transcription in whole blood (tempus) and whole biopsies was assessed. RNA was purified using RNeasy Micro Kit (Qiagen, Courtaboeuf Cedex, France) and quantified using a ND-8000 spectrophotometer (NanoDrop Technologies, Fisher Scientific, Illkirch Cedex, France) before being checked for integrity on the 2100 BioAnalyzer (Agilent Technologies, Massy Cedex, France). In vitro transcription was generated from $200 \mathrm{ng}$ of RNA using AmbionIlluminaTotalPrep RNA Amplification Kits (Applied Biosystems/Ambion, Saint Aubin, France). Labelled cRNA was hybridized onto Illumina Human HT-12v4 BeadChips, The arrays included $>47,000$ probes targeting approximately 36,400 genes, including splice variants and nonannotated gene candidates. Standard quality control thresholds were applied after preprocessing of signal intensity data. All microarray data are MIAME compliant and the raw and normalized data have been deposited in the MIAME compliant database Gene Expression Omnibus (http://www.ncbi.nlm.nih.gov/ geo/, GEO Series accession number GSE39506).

Quantitative PCR analysis. Total RNA was isolated from rectal biopsies by using the Rneasy Micro Kit according to the manufacturer's protocol (Qiagen) and converted to cDNA with Affinity Script QPCR cDNA synthesis kit (Agilent). Quantitative PCR was performed using Brilliant II SYBR GREEN Q-PCR (Agilent) on Mx3005 QPCR Machine (Agilent Technologies). S14 mRNA whose expression was found to be stable among the different group of patients was used as control to normalize each sample. The relative levels of each gene were calculated using the $2^{-\Delta \Delta \mathrm{Ct}}$ method.

The following primers were used (forward/reverse, $5^{\prime}-3^{\prime}$ ):

IL22: AGTTCTGAACAGCTGCACCCACTTC GGGCATCACCT CCTCCAGGTAA

S100A8: GTCCTCAGTATATCAGGAAA ATCAGTGTTGATAT CCAACT

CCR6: AACCAGCTTGCATTTTTTCTGC GAAATTCATTGATT CCCCGCT

S14: GGCAGACCGAGATGAATCCTCA CAGGTCCAGGGGTC TTGGTCC
Total cell-associated HIV-1 DNA quantification in blood and rectal biopsies. The HIV DNA level in peripheral blood mononuclear cells was quantified in whole blood using the ANRS real-time PCR method (Biocentric, Bandol, France), as previously described. ${ }^{47}$ The quantification limit is five copies/PCR. All samples were tested in real time with the same assay throughout the study. Total cell DNA extracts were systematically tested in duplicate and retested in quadruplicate in case of very low levels. Results were calculated using the blood formula and expressed in copy numbers per million peripheral blood mononuclear cells.

Rectal biopsies were stored at $-80^{\circ} \mathrm{C}$ before use. Total HIV DNA was extracted from rectal tissues using a phenol-chloroform protocol, according to the manufacturer's instructions. Total cell-associated HIV-1 DNA was quantified in rectal biopsies by the same ultrasensitive real-time PCR assay.

Statistics. Comparisons between groups were made using the Mann-Whitney $U$-test for continuous data. The Spearman's rank test was used for bivariate correlations, while linear regression analysis was used to produce an accompanying best-fit line. $P$-values of $\leqslant 0.05$ were considered significant.

Gene transcription data were preprocessed ${ }^{48,49}$ and corrected for a chip effect. ${ }^{50}$ Statistical comparisons between the two identified clusters were based on empirical Bayes-moderated $t$-statistics. ${ }^{51}$ An adaptive FDR procedure was used to control for test multiplicity. Hierarchical clustering heatmap analysis of selected probe sets was performed on raw scaling expression using Euclidean distance matrix and Ward's linkage. ${ }^{52}$

Canonical pathway and biological function analyses were then carried out using genes differentially expressed between the two clusters with adaptive FDR-adjusted $P<0.05$ and fold-change $|\mathrm{FC}|>1.5$. Statistical analyses were done with GraphPad Prism Version 5.0a (GraphPad Software, La Jolla, CA) and R (version 3.0.1; The R foundation for Statistical Computing, Vienna, Austria). Ingenuity software (version 12710793; Ingenuity Systems, Redwood City, CA) was used for gene pathway and function analyses. Logistic regression was used to identify factors associated with classification on cluster 1 compared with cluster 2 . Factors investigated were the timing of cART initiation, duration from HIV diagnosis, and overall cART exposure.

SUPPLEMENTARY MATERIAL is linked to the online version of the paper at http://www.nature.com/mi

\section{ACKNOWLEDGMENTS}

We thank the study volunteers for their willingness to participate in this research project. We also thank Barbara De Dieuleveult for managing the study participants. This project was supported by ANRS (Agence Nationale de Recherche contre le SIDA et les hépatites virales). A.K. was supported by a grant from Sidaction and foundation of Pierre Berge. We are grateful to Audrey Riou and Catherine Dehoulle at Service d'Immunologie Biologique, Hôpital Henri Mondor for the preparation of immunohistochemical slides. Technical assistance with microscopy on immunohistochemistry slides was provided by Xavier Decrouy at the plateforme d'imagerie Cellulaire et Tissulaire at INSERM/IMRB/University Paris 12 and by Julien Calderaro at the pathology department at Henri Mondor Hospital. We would like to acknowledge Gabriella Scarlatti and Jean-Daniel Lelièvre for stimulating discussions.

\section{DISCLOSURE}

The authors declared no conflict of interest.

(c) 2015 Society for Mucosal Immunology

\section{REFERENCES}

1. Brenchley, J.M. et al. CD4 + Tcell depletion during all stages of HIV disease occurs predominantly in the gastrointestinal tract. J. Exp. Med. 200, 749-759 (2004) 
2. Dandekar, S., George, M.D. \& Baumler, A.J. Th17 cells, HIV and the gut mucosal barrier. Curr. Opin. HIV AIDS 5, 173-178 (2010).

3. Mehandru, S. et al. Primary HIV-1 infection is associated with preferential depletion of CD4 + Tlymphocytes from effector sites in the gastrointestinal tract. J. Exp. Med. 200, 761-770 (2004).

4. Douek, D.C., Roederer, M. \& Koup, R.A. Emerging concepts in the immunopathogenesis of AIDS. Annu. Rev. Med. 60, 471-484 (2009).

5. Cecchinato, V. \& Franchini, G. Th17 cells in pathogenic simian immunodeficiency virus infection of macaques. Curr. Opin. HIVAIDS 5, 141-145 (2010).

6. Klatt, N.R. et al. Loss of mucosal CD103 + DCs and IL-17 + and IL-22 + lymphocytes is associated with mucosal damage in SIV infection. Mucosal Immunol. 5, 646-657 (2012).

7. Estes, J.D. et al. Damaged intestinal epithelial integrity linked to microbial translocation in pathogenic simian immunodeficiency virus infections. PLoS Pathog. 6, e1001052.

8. Pandya, A.D. et al. Identification of human NK17/NK1 cells. PLoS One 6, e26780 (2011).

9. Spits, H. \& Di Santo, J.P. The expanding family of innate lymphoid cells: regulators and effectors of immunity and tissue remodeling. Nat. Immunol. 12, 21-27 (2011).

10. Cella, M. et al. A human natural killer cell subset provides an innate source of IL-22 for mucosal immunity. Nature 457, 722-725 (2009).

11. Weaver, C.T., Elson, C.O., Fouser, L.A. \& Kolls, J.K. The Th17 pathway and inflammatory diseases of the intestines, lungs, and skin. Annu. Rev. Pathol. 8, 477-512.

12. Pelletier, M. et al. Evidence for a cross-talk between human neutrophils and Th17 cells. Blood 115, 335-343.

13. Favre, D. et al. Critical loss of the balance between Th17 and Tregulatory cell populations in pathogenic SIV infection. PLoS Pathog. 5, e1000295 (2009).

14. Shaw, J.M. et al. Increased frequency of regulatory T cells accompanies increased immune activation in rectal mucosae of HIV-positive noncontrollers. J. Virol. 85, 11422-11434 (2011).

15. Guaraldi, G. et al. Premature age-related comorbidities among HIV-infected persons compared with the general population. Clin. Infect. Dis. 53, 1120-1126.

16. Hunt, P.W. et al. Relationship between T cell activation and CD4 + T cell count in HIV-seropositive individuals with undetectable plasma HIV RNA levels in the absence of therapy. J. Infect. Dis. 197, 126-133 (2008).

17. Hocqueloux, L. et al. Long-term antiretroviral therapy initiated during primary HIV-1 infection is key to achieving both low HIV reservoirs and normal T cell counts. J. Antimicrob. Chemother. 68, 1169-1178 (2013).

18. Hocqueloux, L. et al. Long-term immunovirologic control following antiretroviral therapy interruption in patients treated at the time of primary HIV-1 infection. AIDS 24, 1598-1601 (2010).

19. Saez-Cirion, A. et al. Post-treatment HIV-1 controllers with a long-term virological remission after the interruption of early initiated antiretroviral therapy ANRS VISCONTI Study. PLoS Pathog. 9, e1003211 (2013).

20. Fiebig, E.W. et al. Dynamics of HIV viremia and antibody seroconversion in plasma donors: implications for diagnosis and staging of primary HIV infection. AIDS 17, 1871-1879 (2003).

21. Le Bourhis, L. et al. Mucosal-associated invariant T cells: unconventional development and function. Trends Immunol. 32, 212-218.

22. Brenchley, J.M. et al. Differential Th17 CD4 T-cell depletion in pathogenic and nonpathogenic lentiviral infections. Blood 112, 2826-2835 (2008).

23. Ivanov, I.I. et al. Specific microbiota direct the differentiation of IL-17producing T-helper cells in the mucosa of the small intestine. Cell Host Microbe 4, 337-349 (2008).

24. Favre, D. et al. Tryptophan catabolism by indoleamine 2,3-dioxygenase 1 alters the balance of TH17 to regulatory T cells in HIV disease. Sci. Transl. Med. 2, 32 ra36.

25. Chege, D. et al. Sigmoid Th17 populations, the HIV latent reservoir, and microbial translocation in men on long-term antiretroviral therapy. AIDS 25, 741-749 (2011).

26. Guadalupe, M. et al. Severe CD4 + T-cell depletion in gut lymphoid tissue during primary human immunodeficiency virus type 1 infection and substantial delay in restoration following highly active antiretroviral therapy. J. Virol. 77, 11708-11717 (2003).

27. Macal, M. et al. Effective CD4 + T-cell restoration in gut-associated Iymphoid tissue of HIV-infected patients is associated with enhanced Th17 cells and polyfunctional HIV-specific T-cell responses. Mucosal Immunol. 1, 475-488 (2008).
28. Steele, A.K. et al. Microbial exposure alters HIV-1-induced mucosal CD4 + T cell death pathways Ex vivo. Retrovirology 11, 14 (2014).

29. Dillon, S.M. et al. Human intestinal lamina propria CD1c + dendritic cells display an activated phenotype at steady state and produce IL-23 in response to TLR7/8 stimulation. J. Immunol. 184, 6612-6621 (2010).

30. Petermann, F. et al. Gammadelta T cells enhance autoimmunity by restraining regulatory $T$ cell responses via an interleukin-23-dependent mechanism. Immunity 33, 351-363 (2010).

31. Sutton, C.E. et al. Interleukin-1 and IL-23 induce innate IL-17 production from gammadelta T cells, amplifying Th17 responses and autoimmunity. Immunity 31, 331-341 (2009).

32. Leeansyah, E. et al. Activation, exhaustion, and persistent decline of the antimicrobial MR1-restricted MAIT-cell population in chronic HIV-1 infection. Blood 121, 1124-1135 (2013).

33. Le Bourhis, L. et al. Antimicrobial activity of mucosal-associated invariant $T$ cells. Nat. Immunol. 11, 701-708 (2010).

34. Eyerich, S. etal. Th22 cells represent a distinct human Tcell subset involved in epidermal immunity and remodeling. J. Clin. Invest. 119, 3573-3585 (2009).

35. Trifari, S., Kaplan, C.D., Tran, E.H., Crellin, N.K. \& Spits, H. Identification of a human helper Tcell population that has abundant production of interleukin 22 and is distinct from $T(H)-17, T(H) 1$ and $T(H) 2$ cells. Nat. Immunol. 10 , 864-871 (2009).

36. Kim, C.J. et al. A role for mucosal IL-22 production and Th22 cells in HIVassociated mucosal immunopathogenesis. Mucosal Immunol. 5, 670-680 (2012).

37. Brenchley, J.M. Mucosal immunity in human and simian immunodeficiency lentivirus infections. Mucosal Immunol. 6, 657-665 (2013).

38. Epple, H.J. et al. Mucosal but not peripheral FOXP3 + regulatory Tcells are highly increased in untreated $\mathrm{HIV}$ infection and normalize after suppressive HAART. Blood 108, 3072-3078 (2006).

39. Chevalier, M.F. et al. The Th17/Treg ratio, IL-1RA and SCD14 levels in primary HIV infection predict the T-cell activation set point in the absence of systemic microbial translocation. PLoS Pathog. 9, e1003453 (2013).

40. Lochner, M. et al. Microbiota-induced tertiary lymphoid tissues aggravate inflammatory disease in the absence of RORgammat and LTi cells. J. Exp. Med. 208, 125-134 (2011).

41. Zeng, M., Haase, A.T. \& Schacker, T.W. Lymphoid tissue structure and HIV1 infection: life or death for T cells. Trends Immunol. 33, 306-314 (2012).

42. Klatt, N.R. et al. Probiotic/prebiotic supplementation of antiretrovirals improves gastrointestinal immunity in SIV-infected macaques. J. Clin. Invest. 123, 903-907 (2013).

43. Younas, M. et al. IL-7 modulates in vitro and in vivo human memory $t$ regulatory cell functions through the CD39/ATP axis. J. Immunol. 191, 3161-3168 (2013).

44. Cimbro, R. et al. IL-7 induces expression and activation of integrin alpha4beta7 promoting naive T-cell homing to the intestinal mucosa. Blood 120, 2610-2619 (2012).

45. Marafioti, T. et al. Novel markers of normal and neoplastic human plasmacytoid dendritic cells. Blood 111, 3778-3792 (2008).

46. Paterson, J.C. et al. Labeling of multiple cell markers and mRNA using automated apparatus. Appl. Immunohistochem. Mol. Morphol. 16, 371-381 (2008).

47. Avettand-Fenoel, V. et al. LTR real-time PCR for HIV-1 DNA quantitation in blood cells for early diagnosis in infants born to seropositive mothers treated in HAART area (ANRS CO 01). J. Med. Virol. 81, 217-223 (2009).

48. Bolstad, B.M., Irizarry, R.A., Astrand, M. \& Speed, T.P. A comparison of normalization methods for high density oligonucleotide array data based on variance and bias. Bioinformatics 19, 185-193 (2003).

49. Xie, Y., Wang, X. \& Story, M. Statistical methods of background correction for Illumina BeadArray data. Bioinformatics 25, 751-757 (2009).

50. Johnson, W.E., Li, C. \& Rabinovic, A. Adjusting batch effects in microarray expression data using empirical Bayes methods. Biostatistics 8, 118-127 (2007).

51. Smyth, G.K. Linear models and empirical bayes methods for assessing differential expression in microarray experiments. Stat. Appl. Genet. Mol. Biol. 3, Article3 (2004).

52. Eisen, M.B., Spellman, P.T., Brown, P.O. \& Botstein, D. Cluster analysis and display of genome-wide expression patterns. Proc. Natl. Acad. Sci. USA 95, 14863-14868 (1998). 